\title{
Treatment Outcomes of Anorectal Melanoma
}

\author{
Byung Min Choi, Hyoung Ran Kim, Hae-Ran Yun, Seung Ho Choi ${ }^{1}$, Yong Beom Cho, Hee Cheol Kim, \\ Seong Hyeon Yun, Woo Yong Lee, Ho-Kyung Chun \\ Department of Surgery, Samsung Medical Center, Sungkyunkwan University School of Medicine, Seoul; ${ }^{1}$ Department of Surgery, Samsung \\ Changwon Hospital, Sungkyunkwan University School of Medicine, Changwon, Korea
}

Purpose: An anorectal melanoma (AM) is a very rare tumor. However, sufficient data supporting effective surgical options for the disease do not exist. This retrospective review aimed to analyze treatment outcomes for an AM.

Methods: From June 1999 to December 2008, we retrospectively reviewed a prospectively collected consecutive series of 19 patients who had undergone a surgical resection for an AM at a single institute. Surgical method and clinicopathological factors were analyzed.

Results: The median age was 61.4 years (range, 46 to79 years). Main symptoms were an anal mass, hematochezia, perianal pain, tenesmus, fecal incontinence, and bowel habit change. The average duration of symptoms before diagnosis was 7.8 months (range, 1 to 36 months). S- 100 and HMB- 45 were positive in all patients, even in non-melanin pigmentation. There were 12 abdominoperineal resections (APRs) and 7 wide local excisions (WEs). The APR showed longer overall survival when compared with the WE (64.1 months vs. 10.9 months, $\mathrm{P}<0.001)$. No patients who underwent a WE survived more than 13 months.

Conclusion: A high index of suspicion is necessary to establish the diagnosis for an AM in patients with anal symptoms, and S-100 and HMB-45 can be useful markers for an AM. Even with the small number of cases and the short follow-up, our data suggest that an APR for an AM may provide longer survival than a WE.

Keywords: Anorectal melanoma; Wide excision; Abdominoperineal resection; Immunohistochemical markers

\section{INTRODUCTION}

An anorectal melanoma (AM) has been reported to be a very rare tumor. AM represent only approximately $0.4-0.6 \%$ of all melanoma cases [1-3] and $1 \%$ of anorectal malignant tumors $[4,5]$. Nevertheless, its prognosis is very poor $[5,6]$.

Since AM do not respond well to chemotherapy or radiation therapy, selection of surgical treatment is considered to be important, and the abdominoperineal resection and the wide excision are possible surgical methods $[1,7,8]$. Traditionally, the abdominoperineal resection has been preferred. Nonetheless, recently,

Received: August 31, 2010 Accepted: October 15, 2010

Correspondence to: Seong Hyeon Yun, M.D.

Department of Surgery, Samsung Medical Center, Sungkyunkwan University

School of Medicine, 50 Irwon-dong, Gangnam-gu, Seoul 135-710, Korea

Tel: +82-2-3410-6546, Fax: +82-2-3410-6980

E-mail: shyunmd@skku.edu

(C) 2011 The Korean Society of Coloproctology

This is an open-access article distributed under the terms of the Creative Commons Attribution NonCommercial License (http://creativecommons.org/licenses/by-nc/3.0) which permits unrestricted noncommercial use, distribution, and reproduction in any medium, provided the original work is properly cited. cases treated with only a wide excision have shown outcomes comparable to those achieved when using an abdominoperineal resection $[7,9,10]$. However, studies based on objective data that allow the surgical method to be selected are not sufficient. In our study, the clinical characteristics of an AM were examined, and variations in the treatment outcomes after surgical resection according to the surgery method used were analyzed.

\section{METHODS}

Among a total of 2,753 patients diagnosed as having anorectal malignant tumors from June 1999 to December 2008, 19 patients who were definitely diagnosed histologically as having an AM were selected as the subjects of this study (minimal follow-up period, 7 months). In this retrospective study, the medical records of patients were examined, and the characteristic of patients, surgical methods, prognoses, and pathological records were analyzed. For the comparisons of the clinical characteristics of the two groups and of the survival rate, the chi-square test and the Kaplan-Meier test were used. 


\section{RESULTS}

Among the total 19 patients, 8 patients were males, and 11 patients were females, and their average age was 61.4 years (range, 46 to 79 years). At the time of the visit to our outpatient clinic, common symptoms were rectal tumors (47\%), hematochezia (42\%), and pain in the anal area (16\%). In addition, tenesmus, fecal incontinence, change in bowel habit, etc. were shown. The interval from the onset of symptoms to the diagnosis of an AM was an average of 7.8 months (range, 1 to 36 months).

Among the 19 patients, abdominoperineal resection was performed on 12 patients, and wide excision was performed on $7 \mathrm{pa}$ tients. Among the 12 patients who underwent an abdominoperineal resection, 4 patients were males, and 8 patients were females. Among the 7 patients who underwent a wide excision, 4 patients were males, and 3 patients were females. The mean ages of the two groups were 59 years and 63 years, respectively. The interval from the onset of symptoms to the diagnosis an AM in the abdominoperineal group was an average of 6.9 months (range, 1 to 24 months), and that in the wide excision group was an average of 8.3 months (range, 1 to 36 months). The average tumor size for the abdominoperineal group was $3.9 \mathrm{~cm}$ (range, 0.6 to $4.7 \mathrm{~cm}$ ), which was bigger than the $2.6 \mathrm{~cm}$ (range, 1.2 to $4.5 \mathrm{~cm}$ ) for the wide excision group, but the difference between the two groups was not statistically significant. The average distance from the anal skin to the tumor for the abdominoperineal resection group was $2.2 \mathrm{~cm}$, and that for the wide excision group was $1.6 \mathrm{~cm}$. Melanization was detected in 12 patients $(63 \%)$ of the total 19 patients ( 6 patients in the abdominoperineal resection group, and 6 patients in the wide excision group) (Fig. 1).

In the abdominoperineal resection group, 33\% of the patients (4/12 patients) received postsurgical adjuvant chemotherapy while in the wide excision group in $29 \%$ of the patients ( $2 / 7$ patients) did, and these percentages are not high. Similarly, the percentages of patients receiving postsurgical adjuvant radiation therapy in the

Table 1. Clinical characteristics of two groups (APR and WE)

\begin{tabular}{lcc}
\hline & APR $(\mathrm{n}=12)$ & WE $(\mathrm{n}=7)$ \\
\hline Sex & & \\
$\quad$ Male & $4(33)$ & $4(57)$ \\
$\quad$ Female & $8(67)$ & $3(43)$ \\
Age $(\mathrm{yr})$ & $59(42-79)$ & $63(54-76)$ \\
Time to diagnosis (mo) & $6.9(1-24)$ & $8.3(1-36)$ \\
Tumor size (cm) & $3.9(0.6-4.7)$ & $2.6(1.2-4.5)$ \\
Distance from anal verge $(\mathrm{cm})$ & $2.2(0-10)$ & $1.6(0-3)$ \\
Melanotic discoloration & $6(50)$ & $6(86)$ \\
Postop. chemotherapy & $4(33)$ & $2(29)$ \\
Postop. radiotherapy & $2(17)$ & $2(29)$ \\
\hline
\end{tabular}

Values are presented as number (\%) or value (range).

APR, abdominoperineal resection; WE, wide local excision. two groups were $17 \%$ (2/12 patients) and 29\% (2/7 patients), respectively; thus, postsurgical adjuvant radiation therapy was not performed aggressively (Table 1).

For a pathologically definite diagnosis of melanoma, immunohistochemical staining methods were applied. Among the 19 patients, S-100 and HMB-45 staining were performed on 10 patients (Fig. 2). Among the 10 patients, 5 patients showed melanization, and the remaining 5 patients did not show melanization. The results showed that all patients were positive, and even in patients without melanization, positive reactions were detected in immunohistochemical staining.

In the comparison of the survival period after surgery, the mean survival period of the abdominoperineal resection group was 66.1 months (range, 9 to 103 months) while that of the wide excision group was 11.2 months (range, 7 to 13 months). The survival period of the abdominoperineal resection group was shown to be significantly longer $(\mathrm{P}=0.001)$ (Table 2 ).

In the abdominoperineal group, $50 \%$ of the patients survived for longer than 5 years. On the other hand, in the group that only underwent a wide excision, including 1 patient who underwent palliative surgery because of multicentric lung and liver metastases, none of the patients survived for longer than 13 months (Fig. 3).

\section{DISCUSSION}

An AM is a rare disease [1-3]. In Korea, only a few cases and small scale studies have been reported [11-13]. At our institution, patients who underwent surgery for anal and rectal cancer from

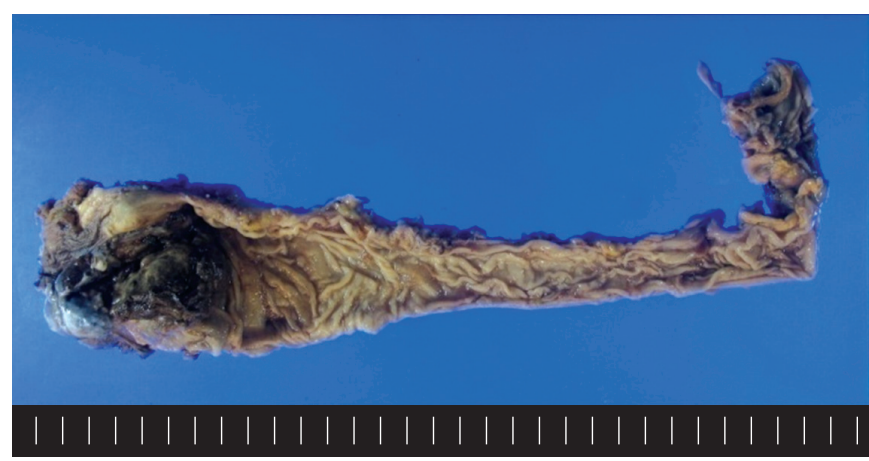

Fig. 1. Gross finding of anorectal malignant melanoma.

Table 2. Comparison of survival between two groups (APR and WE)

\begin{tabular}{lcc}
\hline & APR $(\mathrm{n}=12)$ & WE $(\mathrm{n}=7)$ \\
\hline Overall survival (mo, range) & $66.1(9-103)$ & $11.2(7-13)$ \\
Patient status $(\mathrm{n})$ & & \\
Alive without disease & 6 & 2 \\
Alive with disease & 2 & 0 \\
Dead & 4 & 5 \\
\hline
\end{tabular}

APR, abdominoperineal resection; WE, wide local excision. 

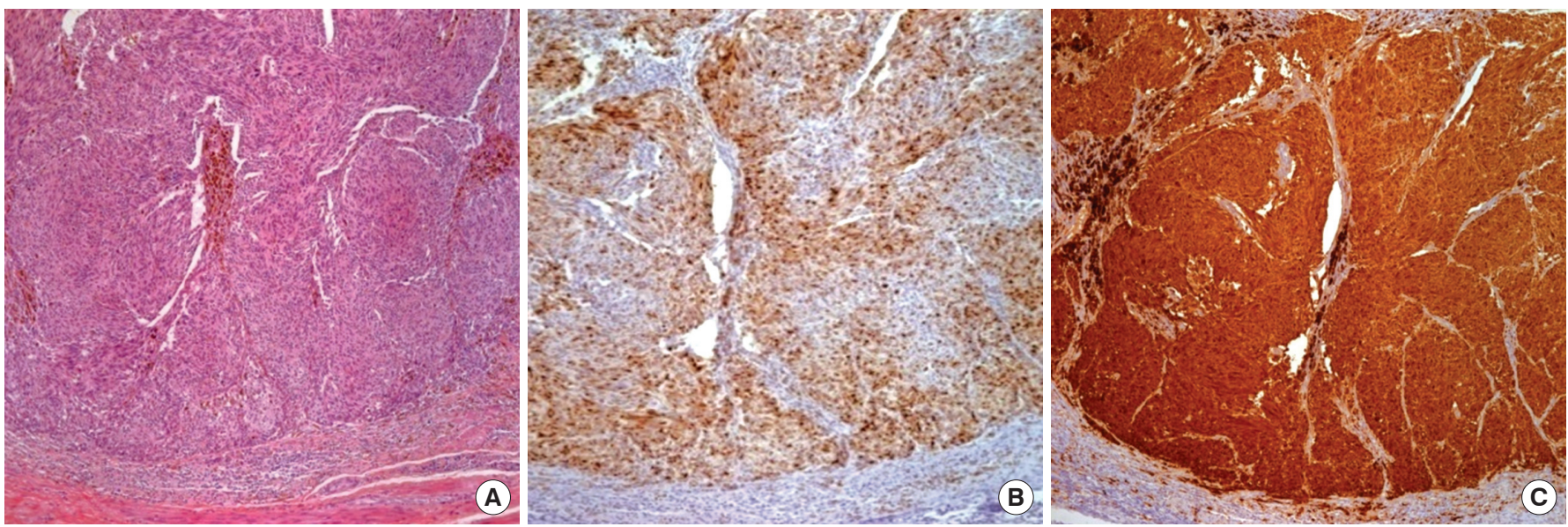

Fig. 2. (A) Pathologic finding of anorectal malignant melanoma (H\&E, $\times 100)$. (B) Pathologic finding of anorectal malignant melanoma (HMB$45, \times 100)$. (C) Pathologic finding of anorectal malignant melanoma (S-100, $\times 100)$.

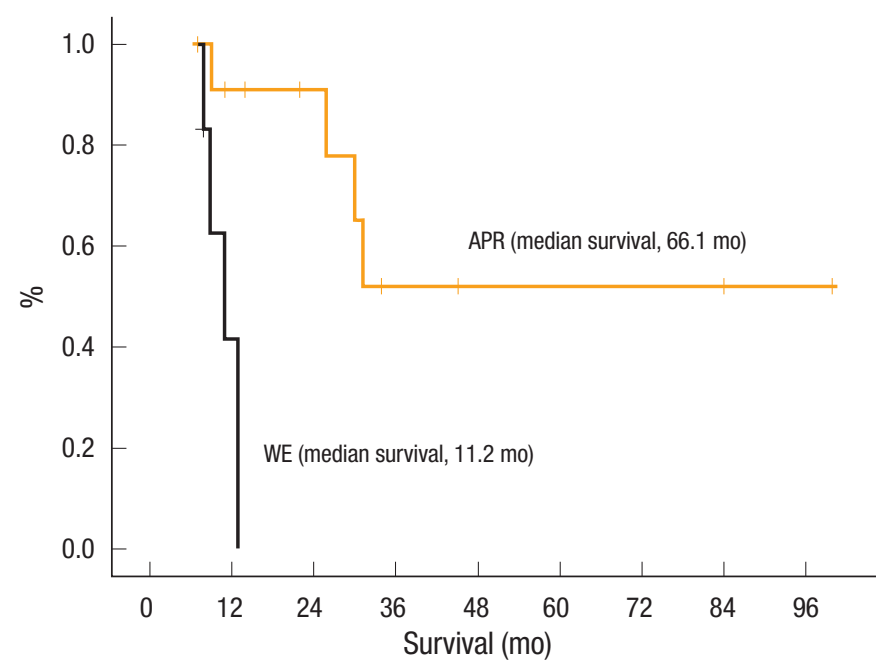

Fig. 3. Survival curves of two groups (APR and WE) $(\mathrm{P}=0.001)$. APR, abdominoperineal resection; WE, wide local excision.

1999 to 2008 totaled 2,753 cases. Among them, only 19 patients were definitely diagnosed pathohistologically as having a melanoma. Because of the small number of patients, it was difficult to validate the statistical significance of the classification of the presurgical clinical characteristics of patients or to compare outcomes according to surgical methods.

In other studies, similarly $[1,14,15]$, symptoms that patients presented with most frequently were tumors in the anal area, hematochezia, and pain in the anal area; hence, the patients were misdiagnosed as having a hemorrhoid. Thus, their conditions were not taken to be serious, and treatments were postponed. In addition, at small hospitals, only partial resections were performed in many cases [14]. Therefore, the interval to the initial diagnosis was long, and the AM was not diagnosed appropriately in many cases. This is associated with the poor prognosis of AM [11]. Therefore, when tumors in the anal area are resected, pathohistological examinations should be performed, and based on the results, appropriate additional treatments should be administered if required.

It has been reported that to determine properly the type of surgery for an AM, the age of the patient, the symptoms, and the general conditions, such as the past history, the size and location of the tumor, and the presence or absence of distant metastasis, should be considered [16]; nonetheless, an objective guideline that could help select the surgical method has not been established yet. In our study, similarly, even though a tendency existed to perform a wide excision for cases in which tumors were located close to the anus, cases with small-sized tumors, and cases with relatively mild symptoms, the preferences of patients themselves and/ or surgeons were added; thus, an objective indication for surgery could not be shown. Treatment guidelines for patients diagnosed as having an AM must be established through continuous studies in the future and through collaboration with other institutions.

Melanization, which has been considered to be specific for the diagnosis of melanoma in the past $[7,15]$, was detected in only $63 \%$ of the cases (12/19 patients). In macroscopic or endoscopic examination, it should be understood that even cases without melanization could be diagnosed as having a melanoma $[7,15]$. In this study, in the pathological diagnosis process, immunohistochemical staining using HMB-45 and S-100 was performed on 10 patients, and regardless of melanization, all cases were found to be positive [17]. HMB-45 melanoma-related antigen is a marker specific to the diagnosis of a melanoma. S-100 protein is a differentiation marker of melanocytes, and its sensitivity has been reported to be high. In addition, mela-A, tyrosinase, and other markers have been used [18]. In the future, use of the immunohistochemical staining technique is recommended for the diagnosis of a melanoma.

In our study, when the survival periods of the patient groups according to surgical methods were compared, all patients surviving 
for longer than 5 years were patients in the abdominoperneal resection group, and this result was statistically significant. Thus, except for patients whose condition is too poor to perform aggressive surgery, an abdominoperineal resection is advantageous for long-term survival.

To accurately diagnose of an AM and to avoid misdiagnose of patients with anal tumors, hematochezia, pain in the anal area and other symptoms as hemorrhoids or other benign diseases in the anal area, pathohistological tests should be performed. Immunohistochemical staining using markers useful for the diagnosis of melanoma HMB-45 and S-100 is recommended. Although this study was conducted on a small number of patients and the follow up was short, as a surgical treatment for an AM, the abdominoperineal resection showed a longer survival than the wide excision.

\section{CONFLICT OF INTEREST}

No potential conflict of interest relevant to this article was reported.

\section{REFERENCES}

1. Goldman S, Glimelius B, Pahlman L. Anorectal malignant melanoma in Sweden: report of 49 patients. Dis Colon Rectum 1990; 33:874-7.

2. Mills SE, Cooper PH. Malignant melanoma of the digestive system. Pathol Annu 1983;18 Pt 2:1-26.

3. Wanebo HJ, Woodruff JM, Farr GH, Quan SH. Anorectal melanoma. Cancer 1981;47:1891-900.

4. Longo WE, Vernava AM 3rd, Wade TP, Coplin MA, Virgo KS, Johnson FE. Rare anal canal cancers in the U.S. veteran: patterns of disease and results of treatment. Am Surg 1995;61:495-500.

5. Weinstock MA. Epidemiology and prognosis of anorectal melanoma. Gastroenterology 1993;104:174-8.

6. Antoniuk PM, Tjandra JJ, Webb BW, Petras RE, Milsom JW, Fazio VW. Anorectal malignant melanoma has a poor prognosis. Int J
Colorectal Dis 1993;8:81-6.

7. Brady MS, Kavolius JP, Quan SH. Anorectal melanoma: a 64-year experience at Memorial Sloan-Kettering Cancer Center. Dis Colon Rectum 1995;38:146-51.

8. Chiu YS, Unni KK, Beart RW Jr. Malignant melanoma of the anorectum. Dis Colon Rectum 1980;23:122-4.

9. Ross M, Pezzi C, Pezzi T, Meurer D, Hickey R, Balch C. Patterns of failure in anorectal melanoma: a guide to surgical therapy. Arch Surg 1990;125:313-6.

10. Ward MW, Romano G, Nicholls RJ. The surgical treatment of anorectal malignant melanoma. Br J Surg 1986;73:68-9.

11. Kim DW, Kang SB, Heo SC, Park KJ, Bang YJ, Park JG. Malignant melanoma of the anorectal region. J Korean Soc Coloproctol 2002; 18:257-61.

12. Lee KH, Koh SH, Hong SW, Yoon C, Joo HZ. Anal malignant melanoma. J Korean Soc Coloproctol 1989;5:33-8.

13. Kim BT, Kim WY, Hwang YH, Rim KS, Shun DJ, Lee KC. A case of rectal malignant melanoma. Korean J Gastroenterol 1985;17: 289-92.

14. Slingluff CL Jr, Vollmer RT, Seigler HF. Anorectal melanoma: clinical characteristics and results of surgical management in twentyfour patients. Surgery 1990;107:1-9.

15. Cooper PH, Mills SE, Allen MS Jr. Malignant melanoma of the anus: report of 12 patients and analysis of 255 additional cases. Dis Colon Rectum 1982;25:693-703.

16. Droesch JT, Flum DR, Mann GN. Wide local excision or abdominoperineal resection as the initial treatment for anorectal melanoma? Am J Surg 2005;189:446-9.

17. Ben-Izhak O, Levy R, Weill S, Groisman G, Cohen H, Stajerman S, et al. Anorectal malignant melanoma: a clinicopathologic study, including immunohistochemistry and DNA flow cytometry. Cancer 1997;79:18-25.

18. Blessing K, Sanders DS, Grant JJ. Comparison of immunohistochemical staining of the novel antibody melan-A with S100 protein and HMB-45 in malignant melanoma and melanoma variants. Histopathology 1998;32:139-46. 